\title{
k-7. Surgical Treatment of Whiplash Injury with Special Reference to Barré-Liéou Syndrome
}

\author{
Yoshito KIRITA \\ Department of Orthopedic Surgery, Tenri Hospital
}

The author has strongly suggested that vertebral instability between the 3rd and 4th cervical bodies is one of the principal causes for the development of Barré-Liéou Syndrome associated with whiplash injury. Based on this concept, anterior cervical spine fusion has been performed in 34 patients, including 24 of whiplash injury and 8 of cervical contusion. Of 19 cases with Barré-Liéou Syndrome six have been treated by $C_{3}-C_{4}$ spine fusion, ten by $C_{3}-C_{5}$ fusion ( 2 intervertebral fixations) and the remaining three by $\mathrm{C}_{3}-\mathrm{C}_{6}$ fusion ( 3 intervertebral fixations). Also, 2 and 3 intervertebral fixations have been performed respectively in two and ten patients of Barré-Liéou Syndrome showing pain radiating to the upper extremities. Of the other three cases with obstinate headache two and the remaining one have had 2 and 3 intervertebral fixations respectively.

A statistical observation has been made on the above cases, regarding early post-traumatic symptoms, time interval between the injury and the operation, and preoperative complaints.

The operative results include complete recovery in 21 and relative relief in 4 instances while 7 are being followed up.

It is concluded that spine fusion of the 3 rd and 4 th cervical bodies is a very effective treatment for Barré-Liéou Syndrome. Postoperative cervical functions are also described.

\section{Discussion to k-7.}

\author{
Hajime Handa, Kohzo Yoshida, Shinsuke Tominaga, Hideo Satoh, \\ Michio Nishikawa and Satoshi Shimizu \\ Department Neurosurgery, Kyoto University Medical School
}

The purpose of this study is to call attention to a form of dizziness of cervical origin following whiplash injury that is precipitated by changes in position of the head, associated with the instability of the upper cervical spines and not found out norrowing of the vertebrobasilar arterial system in an arteriographic study.

It was not relieved by block of the stellate ganglion in the cervical region, but prevented by orthopedic cervical collar.

That an effect on dizziness is produced by an orthopedic collar would 
suggest some indication for interbody fusion of the upper, instable, part of the cervical spine.

\title{
k-8. Surgical Treatment of Barré-Léiou Syndrome due to whiplash injury
}

\author{
Osamu Kataoka, Akira Kurihara, Hideo Watanabe and Fukko Ryo \\ Department of Orthopedic Surgery, Kobe University School of Medicine
}

Most whiplash injured cases are cured by conservative treatment. However some disabling cases remain. Most of these disabling cases have head, eye, ear symptoms. The pathology of these symptoms (Barré-Liéou syndrome or bulbopontine symptoms) due to whiplash injury are not completely clarified. The pathogenesis concerning the disabling case and its surgical treatment are presented.

The sinu-vertebral nerve (recurrent meningeal nerve), which includes sympathetic fibers and has nerve endings into the disc, posterior longitudinal ligament and epidural tissue, has connection with the posterior cervical sympathetic system consisting of a vertebral nerve and of a superior vertebrobasilar plexus.

It is supposed that traumatic pathologic lesions implicating the cervical spine or other anatomic structures in the cervical region may stimulate elements of the posterior cervical sympathetic system through the sinu-vertebral nerve, and thus which may provoke vasomotor reactions in the vertebrobasilar vascular tree.

Thus injuries of disc and ligamentous structures may responsible for the dysfunction of the autonomic nervous system. Existence of disabling cases due to bulbonpontine lesion, however, is undeniable.

It is our opinion to use surgical treatment of disabling cases due to whiplash injury. The instability of cervical spine resulting from the injury should be stabilized. Forty-four disabling cases which have evident cervical disc lesion as the man cause of dysfunction of autonomic nervous system are treated by anterior disc excision with intervertebral body fusion (Cloward).

In a series of 44 cases, discography, electroencephalography, electromyography, plethysmography, angiography of vertebral artery and mecholyl test for autonomic nerve are performed, and also electronmicroscopic study is done on the removed cervical disc.

The symptoms due to this injury can be effectively relieved by this procedure on the upper cervical levels, especially at the levels of $C_{3}-C_{4}$ and $C_{4}-C_{5}$.

\section{CONCLUSIONS}

1. Sinu-vertebral nerve and posterior cervical sympathetic system are responsible for the onset of disabling cases of whiplash injuries of cervical spine.

2. Traumatic pathologic lesions implicating the cervical structures may cause instability and thus may stimulate elements of the posterior cervical sympathe- 